\title{
PURIFICATION OF GAS EMISSIONS FROM THERMAL POWER PLANTS BY MEANS OF APPARATUS WITH JET-BUBBLING CONTACT DEVICES
}

\author{
Ilnur N. Madyshev ${ }^{1, *}$, Oksana S. Dmitrieva ${ }^{2}$, and Andrey V. Dmitriev ${ }^{2}$ \\ ${ }^{1}$ Kazan National Research Technological University, 420016 Kazan, Russia \\ ${ }^{2}$ Kazan State Power Engineering University, 420066 Kazan, Russia
}

\begin{abstract}
A jet-bubble contact device is designed to enhance the operation efficiency of heat and mass exchange units. New devices should be introduced for the contact of gas and liquid, which can provide the minimum entrainment of liquid by the gas flow and have a high phase contact area and relatively low hydraulic resistance. Results of study of a resistance of the dry layer of contact elements at design parameters of jetbubbling contact devices have been presented. Influence of average gas consumption rate on the specific hydraulic resistance of jet-bubbling contact devices has been analyzed.
\end{abstract}

\section{Introduction}

According to the data of The Energy Research Institute (The Russian Academy of Sciences), they expect the industrial growth and energy consumption by 35-70\%, depending on the scenario of the electric-power industry development, if there is a significant substitution of oil and gas by renewable resources and coal [1]. This situation implies the development of effective purification methods of gas emissions from thermal power plants (TPP), operating on heavy fuels.

The problem solution of catching the sulphur dioxide and nitrogen oxides out of the flue gases from the thermal power plants is connected with the processing of large volumes of gas emissions, exceeding in some cases million cubic meters per hour. Used for the industrial purposes, the absorption apparatus are eliminated for the purification of gases, because they have low gas throughput rate $(1.5-2.0 \mathrm{~m} / \mathrm{s})$. The increase in their diameter leads to an increase of gas and liquid non-uniformity along the cross section of the apparatus and the significant reduction of the purification efficiency [2].

For the effective purification of industrial large-capacity gas emissions, the use of vortex type devices is very promising, as they have a high throughput rate, intensive interaction of phases and a wide range of stable operation [3-5]. However, significant disadvantages of vortex type devices are high specific energy costs due to high hydraulic resistance.

\footnotetext{
*Corresponding author: ilnyr 91@mail.ru
} 


\section{Jet-bubbling contact device}

To eliminate these disadvantages the authors have developed the jet-bubbling contact device [6], shown in Fig. 1. This device consists of drain cups (down comers), with vertical walls, required to maintain the liquid level inside them. In each row the drain cups are interconnected by means of rods and their top ends are open, while bottom ends (the bottom) have sets of holes, required for the liquid drain on top of the below drain cups.

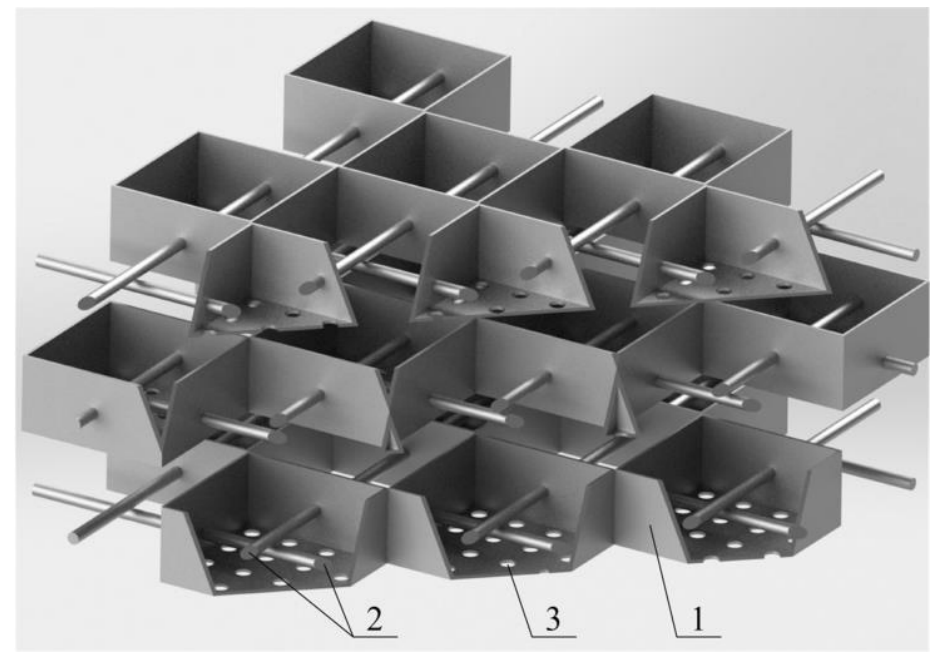

Fig. 1. Jet-bubbling contact device: 1 - drain cup; 2 - rods; 3 - holes.

The jet-bubbling contact device, developed by the authors, operates as follows. The liquid, through the plurality of holes, made in the bottom of the drain cups, is dispersed in the form of jets on top of the below drain cup. Thus, the liquid level in these drain cups, is maintained by the vertical walls of the drain cups. The drain cups are arranged horizontally chequerwise and form a tray. The below tray has an offset of drain cups, forming their vertical chequerwise location. For this reason, the gas, coming from the bottom under the plate, takes on a zigzag movement.

When moving, the liquid jets break up into a multitude of drops. They collide with the liquid surface, inside of the drain cups, and splash every which way. Thus, there is a developed, continuously renewing contact surface of phases, which is determined by the presence of relatively small gas bubbles in the liquid layer and droplets departing from the surface. In addition, the upward gas flow is in contact with the falling drops or liquid jets and, thus, appears the second contact area of gas and liquid.

When the distances between the drain cups are on the same level and equal to the width of the drain cup, it provides stable, uniform gas flow and leads to the reduction of hydraulic resistance of the proposed jet-bubbling contact device. Thus, formation of the original interaction between gas and liquid allows stimulating heat and mass exchange processes both in liquid and in gas phases at relatively simple apparatus design.

\section{Calculation procedure and results}

The purpose of the numerical studies is to determine the hydraulic resistance of the proposed contact devices. For that purpose, all-gas flows through the layer of contact elements were computer simulated via ANSYS software application. In this case, the 
average gas velocity was changing from 1 to $3 \mathrm{~m} / \mathrm{s}$, the width of the contact elements from $0.04 \mathrm{~m}$ to $0.1 \mathrm{~m}$.

The resistance of the dry layer of contact elements can be determined by a modify Darcy-Weisbach equation:

$$
\Delta P_{d r y}=\xi \frac{H}{b} \frac{\rho_{G} W_{G}^{2}}{2},
$$

where $\xi$ - resistance coefficient; $H$ - height of the layer of contact elements, m; $b$ - width of the contact element, $\mathrm{m} ; \rho_{G}-$ gas specific gravity, $\mathrm{\kappa g} / \mathrm{m}^{3} ; W_{G}$ - average gas consumption rate, $\mathrm{m} / \mathrm{s}$.

It should be noted that the resistance coefficient is almost independent of the Reynolds criterion for gas (if $\operatorname{Re}_{G}=1.5 \times 10^{3}-1.8 \times 10^{4}$ ) and of the width of the contact element. Therefore, in engineering calculations it can be taken equal to 9.6, while the relative error does not exceed $\pm 5.2 \%$.

Fig. 2 and Fig. 3 illustrate the dependence of the specific hydraulic resistance of jetbubbling contact devices on the average gas consumption rate and width of the contact element. Fig. 2 shows that the impact of the specific hydraulic resistance on the width of the contact elements is most pronounced at high gas velocities.

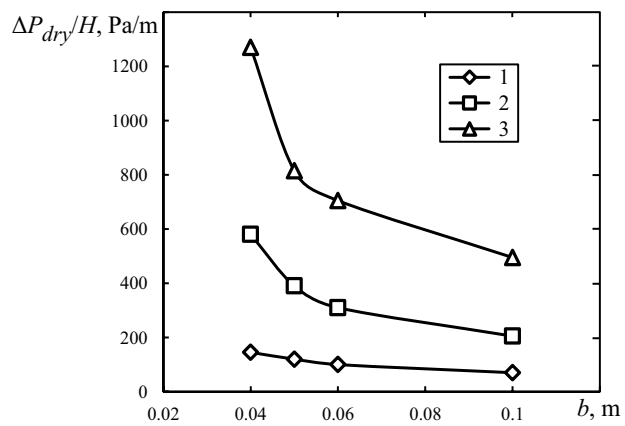

Fig. 2. The specific hydraulic resistance of jetbubbling contact devices, depending on the width of the contact element at different average gas consumption rate $W_{G}, \mathrm{~m} / \mathrm{s}: 1-1 ; 2-2 ; 3-3$.

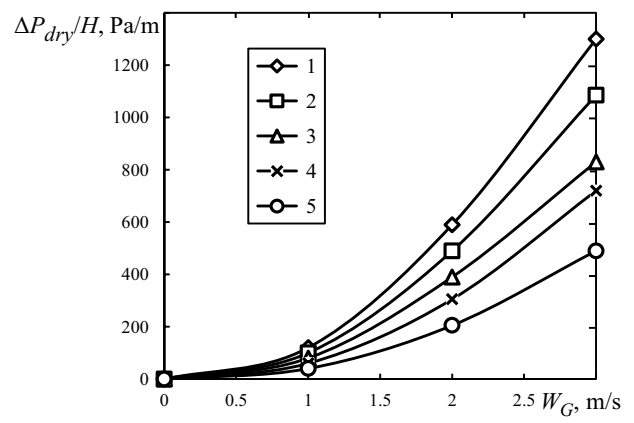

Fig. 3. The specific hydraulic resistance of jetbubbling contact devices, depending on average gas consumption rate at different width of the contact element $b, \mathrm{~m}: 1-0.04 ; 2-0.05 ; 3-$ $0.06 ; 4-0.075 ; 5-0.1$.

Wherein, the increase of the cell width of a contact device leads to the increased average velocity of a fluid flow, which is associated with increasing height of the land which has the greatest speed.

Analyzing the diagrams, it can be concluded that the increase in the width of the contact elements and reduction of the average gas consumption rate leads to a significant reduction of the hydraulic resistance of the proposed contact devices.

\section{Conclusion}

According to the results, the following conclusions can be made:

- if the distance between the drain cups at one level is chosen corresponding to the width of the drain cups, a constant section for the gas flow is provided, which leads to the decrease in the hydraulic resistance of the suggested jet-bubbling contact device, 
- $\quad$ with the increase of the width of elements the average velocity of a flow under any conditions increases,

- developed delivery devices use energy of the liquid, flowing downwards, which reduces the flow resistance for the vapour phase (gas phase),

- thus, it can be stated that the use of the proposed contact devices for purification of gas emissions from thermal power plants, is promising, as they can provide good quality purification at high loads with relatively low energy costs.

The reported study was funded by RFBR, according to the research project No. 16-3860081 mol_a_dk.

\section{References}

1. A. A. Makarov, Science \& technology forecasts and problems of the power engineering development in the Russian Federation up to 2030 79, 206 (2009)

2. A. I. Skoblo, Y. K. Molokanov, A. I. Vladimirov, V. A. Shelkunov, Processes and devices for oil \& gas processing and petrochemical industry (Gubkin Russian State University of Oil and Gas, Moscow, 2007).

3. L. R. Zijatdinova, A. N. Nikolaev, Ecology and industry of Russia, 3, 23 (2009)

4. O. S. Makusheva, A. V. Dmitriev, N. A Nikolaev, Chem. Pet. Eng. 46, 330 (2010)

5. N. A. Voinov, O. P. Zhukova, N. A. Nikolaev, Theor. Found. Chem. Eng. 44, 213 (2010)

6. I. N. Madyshev, O. S. Dmitrieva, A. V. Dmitriev, A. N. Nikolaev, Chem. Pet. Eng. 51, 383 (2015) 